\title{
Editorial
}

\section{Endpoints: The Beginning of a New Treatment?}

\author{
Victor Chong \\ Boehringer Ingelheim International $\mathrm{GmbH}$, Ingelheim am Rhein, Germany
}

When we develop a new treatment for retinal diseases, we think about how to design a clinical trial to show that the new treatment is effective for the targeted retinal diseases. The word "endpoint" is commonly used. In this special issue, we invited a group of experts to write several articles covering different aspects of the topic.

Firstly, Marshall et al. [1] explain the differences in approval between medical devices and pharmaceutical treatments. It is an important subject, as a medical device can gain approval for use without needing to show that it is effective particularly in Europe. Unfortunately, some companies use the term "approved" very loosely for medical devices. For pharmaceutical treatments, the rules are much tighter.

In pharmaceutical development, the sponsor (pharmaceutical company or biotech) demonstrates safety by conducting a phase I study, early efficacy with a phase II, and a phase III study confirms the efficacy, usually by two trials. The two pivotal phase III trials do not have to be identical; indeed, one of the trials can be a large phase II study.

The number of approved retinal drugs in common use are relatively small. Pegaptanib (Macugen) was the first approved anti-VEGF for retinal diseases [2], ranibizumab (Lucentis) and aflibercept (Eylea) are the most commonly used approved retinal drugs. Ranibizumab was approved based on statistically and clinically significant dif-

karger@karger.com

(C) 2021 S. Karger AG, Basel

www.karger.com/oph

Karger! ferences in the proportion of subjects who lose fewer than 15 letters, measured by best-corrected visual acuity (BCVA) at 12 months compared with sham treatment [3]. Similarly, aflibercept was also approved based on clinical equivalence to ranibizumab in the proportion of subjects who lose fewer than 15 letters in BCVA at 12 months [4]. When ranibizumab was approved, patients with exudative age-related macular degeneration (AMD) lost vision very rapidly, so gaining vision was not expected. In the most recent approval of brolucizumab (Beovu), the approval was based on noninferiority to aflibercept in the mean change of BCVA at week 48 , moving away from proportion of subjects who lose fewer than 15 letters [5]. In diabetic macular edema and retinal vein occlusion, the situation is slightly different as these patients do not tend to lose much vision. Hence for approval, a new therapy needs to demonstrate statistically and clinically significant differences in the proportion of subjects who gain more than 15 letters in BCVA [6]. The US Food and Drug Administration (FDA) does not specify the conditions under which the measurement of vision takes place, so in theory, BCVA measured under low luminance can also be used for approval, but so far, no therapy has been approved by this means. This might be important in the development of therapies in conditions that would affect low luminance BCVA more than BCVA, for instance intermediate AMD. 
Geographic atrophy (GA) is another interesting condition regarding approval. At the time of writing, there is no approved treatment for GA; however, in the phase III study of GA by Apellis, the primary endpoint was the reduction in growth of GA lesion size, measured by fundus autofluorescence at month 12 compared to baseline. One can only assume that the FDA and probably other major regulatory agencies have accepted this anatomical endpoint for approval. That is quite critical, as the phase II study of the Apellis compound did not show a benefit for BCVA [7]. Indeed, there are precedents for using anatomical endpoint for approval. Ocriplasmin (Jetrea) was approved based on the proportion of patients with vitreomacular adhesion that had resolved at day 28 after a single intravitreal injection of ocriplasmin as compared to injection of an inactive agent [8].

There is a clear need to treat patients before they lose any vision, such as patients with intermediate AMD. Recently, Wu and Guymer [9] have discussed whether the onset of atrophic AMD can be used as an acceptable endpoint, Mendonca et al. [10] discuss whether the onset of neovascular AMD can be used as an acceptable endpoint and Terheyden et al. [11] discuss the use of composite endpoints for early and intermediate AMD trials.

In patients with less common diseases such as retinal degeneration, the progression of the disease is often slow and variable. The ability to detect small changes and/or a reliable clinical biomarker which can be used for early efficacy signal are important in drug development as later phase clinical trials are very costly. At least in the development of antileakage therapy, the reduction of retinal thickness as measured by optical coherence tomography cannot be used as an approvable endpoint, it is commonly used as an early clinical biomarker of efficacy. In this issue, Stingl et al. [12] introduce new clinical protocols for the evaluation of rod function which might be able to detect early efficacy signals. Similarly, Zollet et al. [13] discuss a novel in vivo technique detecting apoptosis in the retina as a clinical endpoint to be used in early phase clinical development. The FDA previously stated in the setting of glaucoma that a statistically significant difference between groups on pointwise changes of at least $7 \mathrm{~dB}$ may be considered as clinically significant [14]. For a given eye, progression of visual field loss may be defined by five or more visual field locations, which have significant changes from baseline. Yang and Dunbar [15] focus on microperimetry, as microperimetry is more precise than static perimetry, and it can be used as a trial endpoint both for the early detection of efficacy signals and for approval.

Finally, Cheung et al. [16] discuss visual and anatomical endpoints of diabetic macular ischemia, and Wintergerst et al. [17] discuss structural endpoints and outcome measures in uveitis to complete the special issue.

\section{Conflict of Interest Statement}

V.C. is an employee of Boehringer Ingelheim International $\mathrm{GmBH}$; however, the opinion expressed in this paper is not endorsed by Boehringer Ingelheim.

\section{Funding Sources}

No funding was received.

\section{Author Contributions}

V.C. is the sole contributor to this paper.

\section{References}

1 Marshall J, Morrill K, Gobbe M, Blanchard L. The difference between approval processes for drugs and medical devices in Europe. Ophthalmologica. 2021. DOI: 10.1159/000517522.

2 Doggrell SA. Pegaptanib: the first antiangiogenic agent approved for neovascular macular degeneration. Expert Opin Pharmacother. 2005 Jul;6(8):1421-3.

3 Rosenfeld PJ, Brown DM, Heier JS, Boyer DS, Kaiser PK, Chung CY, et al; MARINA Study Group. Ranibizumab for neovascular age-related macular degeneration. N Engl J Med. 2006 Oct;355(14):1419-31.
4 Heier JS, Brown DM, Chong V, Korobelnik JF, Kaiser PK, Nguyen QD, et al; VIEW 1 and VIEW 2 Study Groups. Intravitreal aflibercept (VEGF trap-eye) in wet age-related macular degeneration. Ophthalmology. 2012 Dec;119(12):2537-48.

5 Dugel PU, Koh A, Ogura Y, Jaffe GJ, SchmidtErfurth U, Brown DM, et al; HAWK and HARRIER Study Investigators. HAWK and HARRIER: phase 3, multicenter, randomized, double-masked trials of brolucizumab for neovascular age-related macular degeneration. Ophthalmology. 2020 Jan;127(1):72-84.
6 Boyer DS, Yoon YH, Belfort R Jr, Bandello F, Maturi RK, Augustin AJ, et al; Ozurdex MEAD Study Group. Three-year, randomized, sham-controlled trial of dexamethasone intravitreal implant in patients with diabetic macular edema. Ophthalmology. 2014 Oct; 121(10):1904-14

7 Liao DS, Grossi FV, El Mehdi D, Gerber MR, Brown DM, Heier JS, et al. Complement C3 inhibitor pegcetacoplan for geographic atrophy secondary to age-related macular degeneration: a randomized phase 2 trial. Ophthalmology. 2020 Feb;127(2):186-95. 
8 Stalmans P, Benz MS, Gandorfer A, Kampik A, Girach A, Pakola S, et al; MIVI-TRUST Study Group. Enzymatic vitreolysis with ocriplasmin for vitreomacular traction and macular holes. N Engl J Med. 2012 Aug;367(7):60615.

9 Wu Z, Guymer RH. Can the onset of atrophic age-related degeneration be an acceptable endpoint for preventative trials? Ophthalmologica 2021. DOI: 10.1159/000510887.

10 Mendonca LSM, Levine ES, Waheed NK. Can the onset of neovascular age-related macular degeneration be an acceptable endpoint for prophylactic clinical trials? Ophthalmologica. 2021. DOI: $10.1159 / 000513083$.
11 Terheyden JH, Schmitz-Valckenberg S, Crabb DP, et al. Use of composite end points in early and intermediate age-related macular degeneration clinical trials: state-of-the-art and future directions. Ophthalmologica. 2021. DOI: $10.1159 / 000513591$.

12 Stingl K, Stingl K, Nowomiejska K, et al. Clinical protocols for the evaluation of rod function. Ophthalmologica. 2021. DOI: 10.1159/ 000510888.

13 Zollet P, Yap TE, Cordeiro F. Detecting apoptosis as a Clinical endpoint for proof of a clinical principle. Ophthalmologica. 2021. DOI: $10.1159 / 000513584$.

14 Weinreb RN, Kaufman PL. The glaucoma research community and FDA look to the future: a report from the NEI/FDA CDER Glaucoma Clinical Trial Design and Endpoints Symposium. Invest Ophthalmol Vis Sci. 2009 Apr;50(4):1497-505.
15 Yang Y, Dunbar H. Clinical perspectives and trends: microperimetry as a trial endpoint in retinal disease. Ophthalmologica. 2021. DOI: $10.1159 / 000515148$

16 Cheung CMG, Pearce E, Fenner B, et al. Looking ahead: visual and anatomical endpoints in future trials of diabetic macular ischemia. Ophthalmologica. 2021. DOI: 10.1159/ 000515406.

17 Wintergerst MWM, Liu X, Terheyden JH, et al. Structural endpoints and outcome measures in uveitis. Ophthalmologica. 2021. DOI: $10.1159 / 000517521$. 